\title{
INVESTIGATION OF STRESS-STRAIN STATE OF A WORKPIECE AT GAUGE BURNISHING OF ITS HOLES
}

\author{
Štefan Václav ${ }^{1}$, Nikolay Sergeevich Sivtsev² ${ }^{2}$ Katarína Senderská ${ }^{3}$ \\ 1 Slovak University of Technology, Faculty of Materials Science and Technology, Institute of Production \\ Technologies, Jána Bottu č. 2781/25, 91724 Trnava, Slovakia, e-mail: stefan.vaclav@stuba.sk \\ 2 Kalashnikov Izhevsk State Technical University, Izhevsk, Russia, e-mail: sivcevns@mail.ru \\ ${ }^{3}$ Technical University of Košice, Faculty of Mechanical Engineering, Letná 9, 04200 Košice, Slovakia, e-mail: \\ katarina.senderska@tuke.sk
}

Received: 2017.06.22

Accepted: 2017.08.01

Published: 2017.09.03

\begin{abstract}
The application of finite-element method when investigating the stress-strain state of a workpiece exposed to the gauge burnishing under the conditions of nonzero friction is considered. The mathematical model of the workpiece surface-plastic deformation during burnishing, algorithm and computational results of stresses and deformations are given.
\end{abstract}

Keywords: burnishing, workpiece, gauging, mathematical model, stresses, deformations.

\section{INTRODUCTION}

Burnishing is a simple and high-performance method of gauge treatment of holes method of surface plastic deformation (SPD) [1] implemented at the translational movement of a rigid tool (burnishing tool) in the workpiece hole with tension.

Many researches are dedicated to the investigation of burnishing. As a result of works carried out, it was found that the conditions for obtaining the required quality parameters of the hole are eventually determined by the workpiece stress state, which depends on the scheme of deforming force application and contact friction [2,3]. Adjusting the contact friction by changing the tool kinematics, creating effective ways of lubricant flow to the deformation area, selecting the lubricant composition and other methods, we can achieve significant changes in the stress state and redistribution of deformations, both in the surface layer and the workpiece body $[4,5,6]$. Despite the vast theoretical and experimental material and wide process implementation, there is no unified theory of gauge burnishing $[7,8,9]$. This is mainly explained by the lack of clear idea of stress-strain state (SSS) of the local contact under the conditions of workpiece elastic-plastic deformation with nonzero friction $[10,11]$.

The known attempts of constructing the fundamentals of gauge burnishing with analytical methods $[12,13]$ stay within private empirical concepts and are based on assumptions with which the result accuracy in the solutions obtained do not satisfy the practical needs [14]. This seriously complicates the manufacturing preparation since in each specific case the process application requires the experimental substantiation of the mode providing the set parameters of the hole quality indices $[15,16]$. Due to the high level of computer engineering development, the numerical methods are currently used for taking into account the unconventional features in the calculations of metal-forming processes, in particular, the finite-element method $[17,18,19,20]$. 


\section{MATHEMATICAL MODEL OF THE WORKPIECE SURFACE PLASTIC DEFORMATION DURING BURNISHING BASED ON THE FINITE-ELEMENT METHOD}

The defining system of linear algebraic equations connecting the loads and displacements in the row of discrete points of a solid is written down as follows:

$$
\{\mathbf{W}\}=[\mathbf{K}]\{\delta\}
$$

where: $\delta\}$ and $\{\mathbf{W}\}$ - vectors of nodal displacements and nodal forces; [K] - full matrix of the whole deformed body rigidity.

To obtain a single solution, the system (1) should be complemented with boundary conditions in strains (force method), or in displacements (displacement method), or some of the strains and some of the displacements (mixed problem).
To solve the equation system (1) let us formulate the boundary conditions in a form of nodal force vector as applicable to the workpiece burnishing with nonzero friction (Fig. 1a). For this we introduce the conventional discrete layer with average thickness $\mathrm{H}^{\mathrm{cp}}{ }_{\mathrm{li}}$ in region $1_{\mathrm{i}}$ between two nodal points (Fig. 1b.):

$$
H_{l_{i}}^{\mathrm{av}}=R_{\mathrm{Zw} k p}-\Delta R_{Z_{i}}^{\mathrm{av}}+R_{Z}
$$

where: $R_{Z_{w k p}}$ and $R_{z_{t}}$ - height of microroughnesses of the initial profile of the workpiece and tool surfaces in ten points, respectively; $\Delta R_{z_{i}}^{a v}$ - average value of the workpiece microroughness crushing in $i$ region of the contact surface (the value of the approach of contact surfaces of the tool and workpiece).

Let us take this layer as an integral unit with the tool and transfer nodal points from the surface elements of actual contact of tool and workpiece microroughnesses (external friction surfaces)

a)

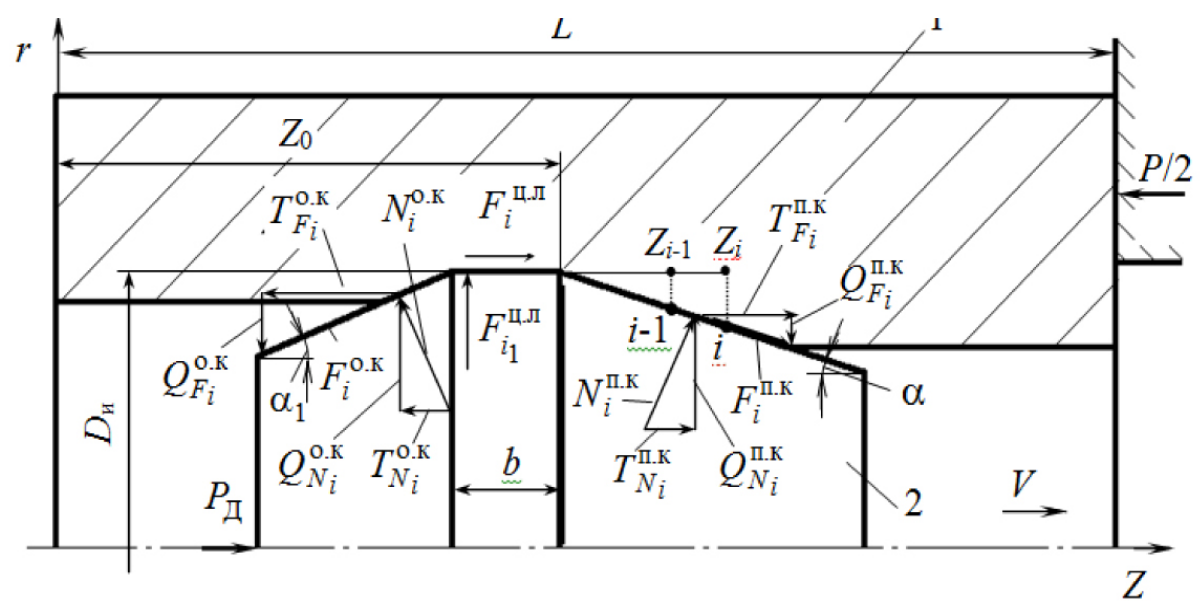

b)

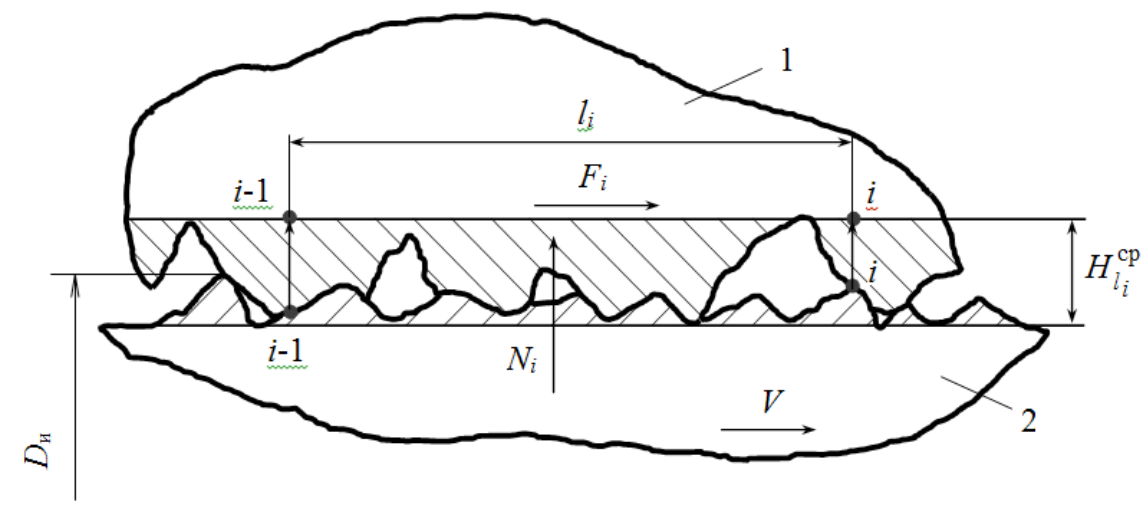

Fig. 1. Scheme of workpiece loading during burnishing with nonzero friction: a) force vectors acting upon the workpiece; b) discrete contact layer between the nodal points; 1 - workpiece; 2 - tool; $D_{N}, \alpha, \alpha_{1}$ and $b$ - nominal diameter, angle of front, inverse cones and width of the tool cylindrical band, respectively; $P$ and $V$ - force and speed of burnishing, respectively; $L$ - workpiece length 
onto the nominal surface (Fig. 1b). Then the areas $S_{N}$ of $i$ regions between the two nodal points are determined as follows:

- on the front cone:

$$
S_{\mathrm{N}_{i}}=\pi\left[r_{i-1}+r_{i}+2\left(R_{Z_{\mathrm{wkp}}}-\Delta R_{Z_{i}}^{\mathrm{av}}\right)\right] \frac{Z_{i}-Z_{i-1}}{\cos \alpha}
$$

- on the cylindrical band:

$$
S_{\mathrm{N}_{i}}=\pi\left[r_{i-1}+r_{i}+2\left(R_{\mathrm{Zw} k p}-\Delta R_{Z_{i}}^{\mathrm{av}}\right)\right]\left(Z_{i}-Z_{i-1}\right)
$$

- on the inverse cone:

$$
S_{\mathrm{N}_{i}}=\pi\left[r_{i-1}+r_{i}+2\left(R_{Z \mathrm{w} k p}-\Delta R_{Z_{i}}^{\mathrm{av}}\right)\right] \frac{Z_{i}-Z_{i-1}}{\cos \alpha_{1}}
$$

where $Z_{i}, Z_{i-1}$ and $r_{l}, r_{i-1}$ - coordinates of $i$ and $i-1$ nodal points on the contact surface in the direction of axis $Z$ and tool radii in these points, respectively.

Let us form the vector of nodal forces in the region of the tool front cone.

The friction force $F_{i}$ on the nominal area $S_{N}$, with the average shear stresses $\tau_{N_{i}}$ on it will be found as follows:

$$
F_{i}=\tau_{\mathrm{N}_{i}} \pi\left[r_{i-1}+r_{i}+2\left(R_{Z_{\mathrm{wlp}}}-\Delta R_{Z_{i}}^{\mathrm{av}}\right)\right] \frac{Z_{i}-Z_{i-1}}{\cos \alpha}
$$

The components of friction forces $Q_{F_{i}}$ and $T_{F_{i}}$ equal $Q_{F_{i}}=F_{i} \cdot \sin \alpha ; T_{F_{i}}=F_{i} \cdot \cos \alpha$, respectively.

To find the normal force $N_{i}$, the dependencies obtained when designing the friction mechanicalmathematical model under the conditions of contact interaction of the workpiece and tool rough surfaces under the mutual influence of slip-line fields in the deformation regions of separate microroughnesses on the nominal area $S_{N}$ are used [1]. To obtain the lateral shear of the tool microroughnesses relatively to the workpiece microroughnesses we have:

$$
\begin{aligned}
& N_{i}=1.285\left(2-\frac{\tau_{\mathrm{s}_{i}}}{k \bar{S}_{i}}\right) \bar{S}_{i} \pi\left[r_{i-1}+r_{i}+2\left(R_{\text {Zwkp }}-\Delta R_{Z_{i}}^{\mathrm{av}}\right)\right] \times \frac{Z_{i}-Z_{i-1}}{\cos \alpha} k \text { for } \bar{S}_{i} \leq n \\
& N_{i}=1.285\left(2-\frac{\tau_{\mathrm{s}_{i}}}{k \bar{S}_{i}}\right)\left[n-(1-n) \ln \left(\frac{1-\bar{S}_{i}}{1-n}\right)\right] \pi\left[r_{i-1}+r_{i}+2\left(R_{\text {Zw } k p}-\Delta R_{Z_{i}}^{\mathrm{av}}\right)\right] \times \\
& \times \frac{Z_{i}-Z_{i-1}}{\cos \alpha} k \text { for } n<\bar{S}_{i} \leq 1
\end{aligned}
$$

where: $-\bar{S}_{i}=S / S_{N_{i}}$-relative area between two nodal points; $k$ - shear constant (plasticity constant); n - ordinate of the junction point of the functions: linear (3) and exponential (4) (by Prandtl field $\mathrm{n}=1 / 2$, Hill field $\mathrm{n}=2 / 3$ ) [2].

The components of the normal force $Q_{N_{i}}$ and $T_{N_{i}}$ on the nominal area $S_{N}$ are following: $Q_{N_{i}}=N_{i} \cdot \cos \alpha$, $T_{N_{i}}=N_{i} \cdot \sin \alpha$.

The total forces on the area $S_{N_{i}}: Q_{i}=Q_{N_{i}}-Q_{F i} ; T_{i}=T_{N_{i}}+T_{F_{i}}$.

Accordingly, the forces on the area $S_{N_{i+1}}=Q_{i+1}=Q_{N_{i+1}}-Q_{F_{i+1}} ; T_{i+1}=T_{N_{i+1}}+T_{F_{i+1}}$.

The nodal forces (load vector) in the point with coordinate $Z_{\mathrm{i}}$ are determined as the arithmetic average of the forces acting on the areas $S_{N}$ and $S_{N+1}: Q_{Z_{i}}=\left(Q_{i}+Q_{i+}\right) / 2 ; T_{Z_{i}}=\left(T_{i}+T_{i+}\right) / 2$.

Similarly, the vector of forces is formed in the regions of the cylindrical band and inverse cone of the tool.

The dependencies for finding the friction force (2) and normal force (3) and (4) on the contact nominal area between the nodal points represent the system of two equations with five unknown variables $-F_{i}, N_{i}, \tau_{N_{i}}, \bar{S}_{i}$ and $\Delta R_{Z_{I}}^{a v}$. Obviously, even when introducing the additional boundary 
conditions and using the numerical methods it is impossible to obtain the closed solution for this system.

To decrease the number of the unknown variables, it is proposed to analytically connect the crushing value of the workpiece microroughnesses (approach value) with the actual contact area through the geometrical parameter of the workpiece rough contact layer.

Quite a lot of solutions for determining such connection have been proposed so far. The bestknown solutions are based on the evaluation of the actual contact area with the help of the supporting surface curve characterizing the material distribution in the rough layer. However, these solutions cannot take into account the specifics of SPD during burnishing that consists in the fact that the supporting surface occurs under the conditions of stochastic structure formation and directed texture formation while flattening and flowing of workpiece surface microroughnesses.

To trace the changes in the supporting surface at SPD, it is proposed to approximate it with the function with the sinusoidal feature, deriving which the supporting surface formation is considered not in connection with stock removal during machining, but relative to the mass transfer of the workpiece contact surface roughnesses during their deformation. In accordance with [3] the following dependence is such a function:

$$
\bar{S}=2\left[1-\sin ^{2} \frac{\pi}{2}\left(1-\Delta R_{Z} / R_{\max }\right)\right] \leq 1
$$

where: $R_{\max }$ - maximum height of the workpiece microroughnesses profile.
Since the relative area of the actual contact between the two nodal points $\bar{S}_{i}$, then after the transformation (5) the average value of microroughnesses crushing in this region is found as follows:

$$
\Delta R_{Z_{i}}^{\mathrm{av}}=R_{\max }\left(1-\frac{2}{\pi} \arcsin \sqrt{1-\bar{S}_{i} / 2}\right)
$$

Taking into account the insignificant length of $i$ region between the two nodal points on the contact, the equation obtained when developing the model of contact interaction of separate microroughnesses is used to find the relative area of the actual contact $\bar{S}_{i}[1]$ :

$$
\bar{S}_{i}=\sqrt{\frac{\tau_{\mathrm{N}_{i}} R_{Z_{\mathrm{t}}}\left(\operatorname{tg} \theta_{\mathrm{T}}+\operatorname{ctg} \gamma\right) \operatorname{ctg} \theta_{\mathrm{wkp}}}{1.6 k R_{Z \mathrm{wkp}}\left(1+\theta_{\mathrm{t}}-\gamma\right) \operatorname{ctg} \theta_{\mathrm{t}}}}
$$

where: $\theta_{t}$ and $\theta_{w k p}$ - halves of the profile angles at the apexes of the tool and workpiece microroughnesses; moreover:

$$
\gamma=\arcsin \frac{\cos \theta_{\mathrm{t}}}{\sqrt{2} \sin \gamma}
$$

Then, considering (7), $\Delta R_{Z_{I}}^{a v}$ in the formula (6) can be expressed as the function $\tau_{N_{i}}$, and equations (2), (3) and (4) are converted in the system with three unknown variables $\left(F_{i}, N_{i}, \tau_{N_{i}}\right)$.

The considered problem of the workpiece deformation at burnishing is rendered as the axisymmetric contact one, so, we observe the equality of vectors of opposite in direction nodal forces in conjugated contact nodes of the workpiece and tool (Fig. 2): $\left\{\mathbf{W}_{1}\right\}=\left\{\mathbf{W}_{2}\right\}$, where $\left\{\mathbf{W}_{1}\right\}$ and $\left\{\mathbf{W}_{2}\right\}$ - vectors of contact nodal forces for the workpiece and tool, respectively.

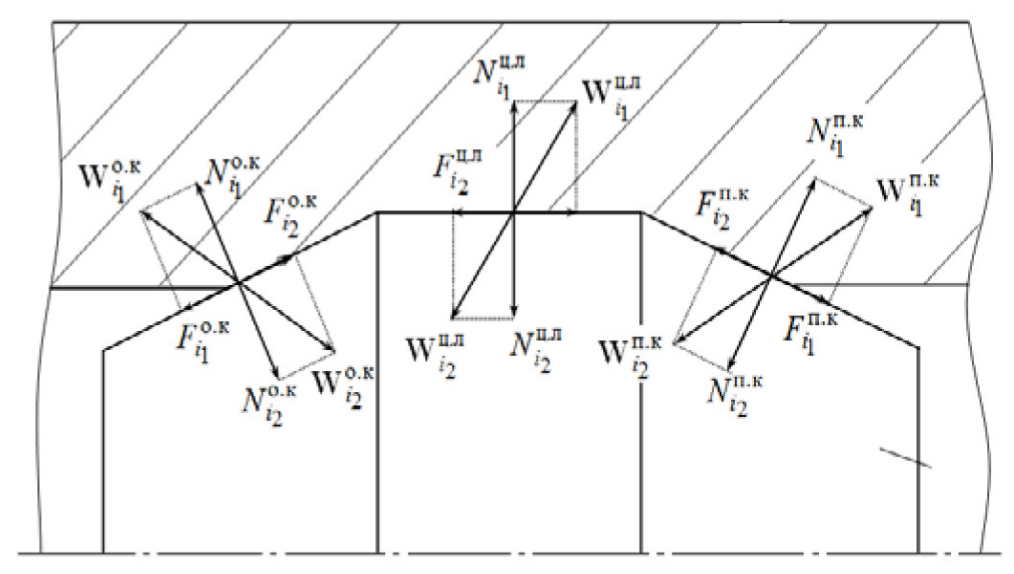

Fig. 2. Vectors of the forces in conjugated contact nodes of the workpiece and tool in the regions of front cone (f.c), cylindrical band (c.b) and inverse cone (i.c): 1 - workpiece; 2 - tool 
The equality of nodal forces on the contact allows, after the formation of two rigidity matrixes (for the workpiece and tool), solving two equation systems in displacements:

$$
\left\{\begin{array}{l}
\left\{\mathbf{W}_{1}\right\}=\left[\mathbf{K}_{1}\right]\left\{\boldsymbol{\delta}_{1}\right\} \\
\left\{\mathbf{W}_{2}\right\}=\left[\mathbf{K}_{2}\right]\left\{\boldsymbol{\delta}_{2}\right\}
\end{array}\right.
$$

where: $\left[\mathbf{K}_{1}\right],\left[\mathbf{K}_{2}\right]$ and $\left\{\boldsymbol{\delta}_{1}\right\},\left\{\boldsymbol{\delta}_{2}\right\}$ - rigidity matrixes and displacement vectors on the contact for the workpiece and tool, respectively.

By its sense, the system (8) corresponds to the mutual consideration of two bodies linked by the condition of displacement compatibility of conjugated nodes on the contact of these bodies. This system can be written down as follows:

$$
\left\{\begin{array}{l}
\left\{\mathbf{r}_{1}\right\}=\left[\mathbf{A}_{1}\right]\left\{\mathbf{W}_{1}\right\} \\
\left\{\mathbf{r}_{2}\right\}=\left[\mathbf{A}_{2}\right]\left\{\mathbf{W}_{2}\right\}
\end{array}\right.
$$

where: $\left[\mathbf{A}_{1}\right]=\left[\mathbf{K}_{1}\right]^{-1},\left[\mathbf{A}_{2}\right]=\left[\mathbf{K}_{2}\right]^{-1}$ and $\left\{\mathbf{r}_{1}\right\},\left\{\mathbf{r}_{2}\right\}$ - inverse rigidity matrixes and vectors of radial displacements for the workpiece and tool, respectively.

Taking into account the discrete contact layer introduced when forming the vector of nodal forces (Fig. 1b), the condition of displacement compatibility in radial direction is as follows:

$$
r_{1_{i}}-r_{2_{i}}=N_{\text {Дi }_{i}} / 2+R_{\max }-\Delta R_{Z_{i}}^{\mathrm{av}}
$$

where: $N_{B_{i}}$ - burnishing interference in i nodal contact point found taking into account the tool geometry.

Due to the undulation of the workpiece surface treated, the burnishing interference in the contact nodal points is found by the formulas:

- in the cylindrical band region:

$$
N_{\mathrm{B}_{i}}=D_{\mathrm{t}}-d_{\mathrm{wkp}}^{\min }-2 W_{Z}\left[1-\cos \frac{2 \pi Z}{S_{W}}\right]
$$

- in the front cone region:

$$
N_{\mathrm{B}_{i}}=D_{\mathrm{t}}-d_{\mathrm{wkp}}^{\min }-2\left\{W_{Z}\left[1-\cos \frac{2 \pi Z}{S_{W}}\right]+\left(Z-Z_{0}\right) \operatorname{tg} \alpha\right\}
$$

where: $d^{\text {min }}{ }_{w k p}-$ minimum diameter of the workpiece hole; $W_{Z}$ and $S_{Z}$ - height and mean a pitch of the undulation of the workpiece surface, respectively.

Equations (9), (10) represent the additional boundary condition in displacements introduced when solving the systems of equations (2), (3) and (4).

To consider the strengthening when solving the problem of elastic-plastic stressing of the workpiece during burnishing, the method of elasticity variable parameters is applied [4], which is based on the use of actual (real) or schematized diagrams of deformation or extension (compression) [5].

\section{ALGORITHM FOR CALCULATING THE WORKPIECE SSS IN THE REGIONS OF THE FRONT CONE AND CYLINDRICAL BAND OF THE TOOL}

Based on the boundary conditions formed, the algorithm for calculating the workpiece SSS during the gauge burnishing of holes in it is developed. The algorithm is based on the variant of implementing the finite-element method discussed in [6].

1. Array of the initial data is formed, including:

- workpiece geometrical parameters (length L, minimum $d^{\min }{ }_{w k p}$ and maximum $d^{\max }{ }_{w k p}$ diameters of the hole treated, external diameter $D_{w k p}$, etc.);

- physical and mechanical parameters of the workpiece material (yield stress $\sigma_{S_{I}}$, elasticity modulus $E_{I}$ and Poisson ratio $\left.\mu_{1}\right)$; 
- workpiece surface parameters (height of microroughnesses of the initial profile in 10 points $R_{Z_{w k p}}$, maximum height of microroughnesses $R_{\max }$, angle at the apex of microroughnesses $2 \theta_{w k p}$, mean pitch $S_{W}$ and surface undulation height $W_{z}$;

- tool geometric parameters (diameter of gauge band $D_{t}$, angle of front $\alpha$ and inverse $\alpha_{l}$ cones, width $b$ of cylindrical band, region length of the front $B$ and inverse $B_{1}$ cones);

- physical and mechanical parameters of the tool material (yield stress $\sigma_{S_{2}}$, elasticity modulus $E_{2}$ and Poisson ratio $\left.\mu_{2}\right)$;

- tool surface parameters (height of microroughnesses of the initial profile in 10 points $R_{Z_{i}}$, angle at the apex of microroughnesses $2 \theta_{t}$ ).

2. Workpiece and tool are divided into axisymmetric finite elements.

3. Properties of the workpiece and tool elements are introduced (matrixes of differentiation of displacements $\left[\mathbf{B}_{1}\right],\left[\mathbf{B}_{2}\right]$ and elasticity $\left.\left[\mathbf{D}_{1}\right],\left[\mathbf{D}_{2}\right]\right)$.

4. Burnishing interference $N_{B_{i}}$ is found in each nodal contact point taking into account the tool geometry and workpiece surface undulation (11), (12).

5. Rigidity matrix of workpiece $\left[\mathbf{K}_{1}\right]$ and tool $\left[\mathbf{K}_{2}\right]$ is formed.

6. Taking into account the boundary conditions formed by the nodal forces, the model of developing the actual contact (5) and proposed model of the contact interaction (7), the nodal displacements are calculated $\left\{\mathbf{r}_{1}\right\},\left\{\mathbf{r}_{2}\right\}$ (9).

7. Correspondence of the nodal displacements to the condition of displacement compatibility is checked (10). If the value of the displacement residual error $\xi$ exceeds $0.0005 \cdot N_{B_{i}}$, the contact area nodal forces are refined. At the same time, the value of the node force incrementation in the axial direction is found by the formula [6]:

$$
\Delta T_{Z_{i_{n}}}=\frac{T_{Z_{i_{n-1}}}-\xi_{i_{n-1}} \cdot 10^{\operatorname{lq}_{Z_{i_{n-1}}}-1}}{\xi_{i_{n}}-\xi_{i_{n-1}}} \cdot\left(\xi_{i_{n-1}}-0.0005 N_{\mathrm{B}_{i}}\right)
$$

where: $\mathrm{T}_{\text {Zin }}, \xi_{\mathrm{in}}$ - axial component of the nodal force and residual error on $\mathrm{n}$ iteration; $\mathrm{T}_{\mathrm{Zin}-1}, \xi_{\mathrm{in}-1}-$ axial component of the nodal force and residual error on $\mathrm{n}-1$ iteration.

Improved axial and radial components of the nodal force on arbitrary iteration equal as follows:

$$
T_{Z_{i_{n}}}=T_{Z_{i_{n-1}}}+\Delta T_{Z_{i_{n}} \text { and }} Q_{Z_{i_{n}}}=f\left(T_{Z_{i_{n}}}\right)
$$

When executing the conditions (10), the refinement of the nodal forces is complete.

8. Deformations $\{\boldsymbol{\varepsilon}\}=[\mathbf{B}]\{\mathbf{a}\}$ and strains $\{\boldsymbol{\sigma}\}=$ $[\mathbf{D}]\{\boldsymbol{\varepsilon}\}=[\mathbf{D}][\mathbf{B}]\{\mathbf{a}\}$ are determined, here $\{\mathbf{a}\}$ - vector of generalized displacement, as well as the intensity of deformations and strains for each element of the workpiece. Then the workpiece rigidity matrix is improved $\left[\mathbf{K}_{\mathbf{1}}\right]$. The calculations are performed until $\Delta E_{l}$ and $\Delta \mu_{1}$ exceed $1 \%$.

\section{RESULTS OF NUMERICAL INVESTIGATION OF THE WORKPIECE SSS}

In accordance with the envisaged plan (as shows Table 1) the stress condition of cylindrical workpieces with the nominal hole diameter $d_{w k p}$ $=45 \mathrm{~mm}$, length $L=40 \mathrm{~mm}$, external diameter $D_{w k p}$ from $54 \mathrm{~mm}$ up to $112 \mathrm{~mm}$ was investigated. The roughness and undulation parameters of the surface treated were the following: $R_{Z_{w k p}}=3.2$ $\mu \mathrm{m}, R_{\text {max }}=1.15 \mu \mathrm{m}, \theta_{w k p}=80^{\circ}, S_{W}=7 \mathrm{~mm}, W_{Z}$ $=6.5 \mu \mathrm{m}$. Workpiece material - steel 45 (GOST (State Standard) 1050-74) after normalization ( $\sigma_{s}$ $=360 \mathrm{MPa}, E=206250 \mathrm{MPa}, \mu=0.3)$.

The angle of the front $\alpha$ and inverse $\alpha_{I}$ cones of the tool varied from $0.5^{\circ}$ up to $20^{\circ}$, the width of cylindrical band $b$ changes from 1 up to $3 \mathrm{~mm}$. The roughness of the tool work surface $-R_{z}=$ $0.8 \mu \mathrm{m}$, internal diameter $-22 \mathrm{~mm}$. The burnishing maximum interference $N_{B}=0.28 \mathrm{~mm}$ was selected from the condition of elastic-plastic deformations emerging in the workpieces.

The calculations were carried out for five different positions of the tool in the hole treated (Fig. 3). The maximum interference of burnishing was selected from the condition of elastic-plastic deformations emerging in the workpieces (deformation degree did not exceed $1 \%$ ). The computational results were 
Table 1. Plan of numerical investigation of the workpiece SSS during and after burnishing

\begin{tabular}{|c|c|c|c|c|c|c|c|c|}
\hline \multirow{2}{*}{$\begin{array}{l}\text { No. of the } \\
\text { trial series }\end{array}$} & \multicolumn{8}{|c|}{ Burnishing process factors investigated and their values } \\
\hline & $f$ & $N_{B}, \mathrm{~mm}$ & $\alpha, \operatorname{deg}$ & $\alpha_{1}$, deg & $b, \mathrm{~mm}$ & $D_{w k p} / d_{w k p}$ & $\sigma_{\mathrm{s}}, \mathrm{MPa}$ & $L, \mathrm{~mm}$ \\
\hline 1. & $0-0.26$ & $\begin{array}{c}0.08 ; 0.12 \\
0.2\end{array}$ & 3 & 10 & 2 & 1.64 & 360 & 40 \\
\hline 2. & $\begin{array}{c}0 ; 0.02 ; 0.04 \\
0.06 ; 0.08 \\
0.10 ; 0.12 ; \\
0.26\end{array}$ & $0.08-0.28$ & 3 & 10 & 2 & 1.64 & 360 & 40 \\
\hline 3. & $0.08 ; 0.12$ & $0.08 ; 0.12$ & $0.5-20$ & 10 & 2 & 1.64 & 360 & 40 \\
\hline 4. & $0.08 ; 0.12$ & $0.08 ; 0.12$ & 3 & $0.5-20$ & 2 & 1.64 & 360 & 40 \\
\hline 5. & $0 ; 0.12 ; 0.26$ & 0.08 & $1 ; 3$ & 10 & $1-3$ & 1.64 & 360 & 40 \\
\hline 6. & $0.08 ; 0.12$ & 0.08 & 3 & 10 & 2 & $1.2-1.64$ & 360 & 40 \\
\hline 7. & $0.08 ; 0,12$ & 0.12 & 3 & 10 & 2 & 1.64 & $\begin{array}{l}250 ; 360 \\
650 ; 800\end{array}$ & 40 \\
\hline \multicolumn{9}{|c|}{$\begin{array}{l}\text { Note: The values of the variable factors are selected taking into account the possibilities of comparing the results of numerical calculations } \\
\text { with the data obtained before in experimental and theoretical investigations of the process }[5-9] ; f-\text { friction coefficient; } N_{B}-\text { burnishing } \\
\text { interference; } \alpha, \alpha_{1} \text { and } b \text {-angle of front, inverse cones and width of cylindrical band of the tool, respectively; } D_{w k p}, d_{w k p} \text { and } L-\text { external, } \\
\text { internal diameters and length of the workpiece; } \sigma_{\mathrm{s}}-\text { yield stress of the material treated. }\end{array}$} \\
\hline
\end{tabular}

compared with the experimental data previously obtained in the investigations of the burnishing process [7-11].

The analysis of the results obtained demonstrated that the maximum axis $\sigma_{z}$, radial $\sigma_{r}$ and circumferential $\sigma_{\theta}$ stresses in the workpiece emerge in the region of its contact with the tool cylindrical band (Figure 4). Here the maximum intensity of stresses $\sigma_{i}$ appears as well.

Despite the tool position in the hole treated in the annular sections, which divide the front and inverse cones of the tool with its cylindrical band, the significant elevation of the stress level is observed (Fig. 4). With the increase in the width of

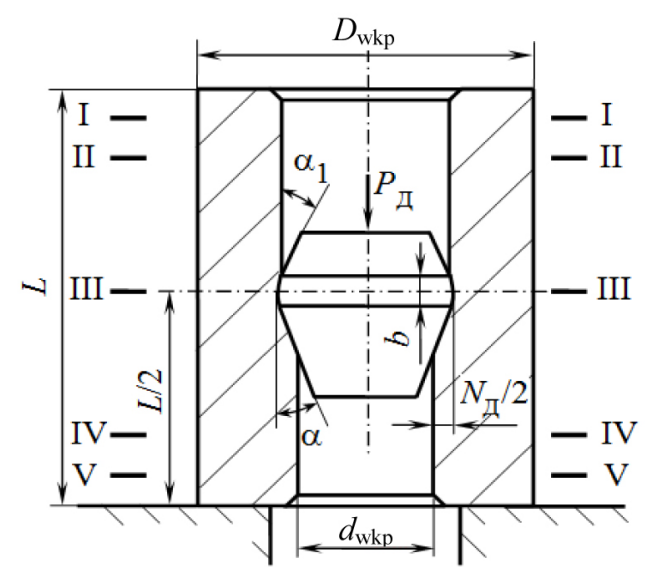

Fig. 3. Radial sections (I-V) investigated in the numerical experiment the cylindrical band and radius of its transition to the conical regions the stress level decreases, thus demonstrating the dominant role of the tool geometric parameters indicated onto the stress state of the burnished workpiece.

The pattern of distribution of radial stresses along the workpiece wall thickness (Figure 5) is in accordance with the previously obtained one based on the analytical methods of solving the known problem on loading a heavy-wall pipe by uniformly distributed internal pressure and axial force. From the maximum compressing on the internal surface they decrease to zero on the external one. The axial stresses, being compressing on the external surface, change their index along the workpiece wall. The tensile circumferential stresses along the workpiece wall do not change the index.

The maximum intensity of stresses for a given material is determined by its ability to deformation strengthening and depends on interference, tool geometry and workpiece wall thickness. It emerges in the surface layer (Figures 4 and 5), where the maximum increase in microhardness after burnishing is observed.

Having reached its maximum in the surface layer, the stress intensity goes down. The nodal point, in which its value equals the initial yield stress of the material treated (Fig. 5), determines the depth of the strengthened surface layer. 

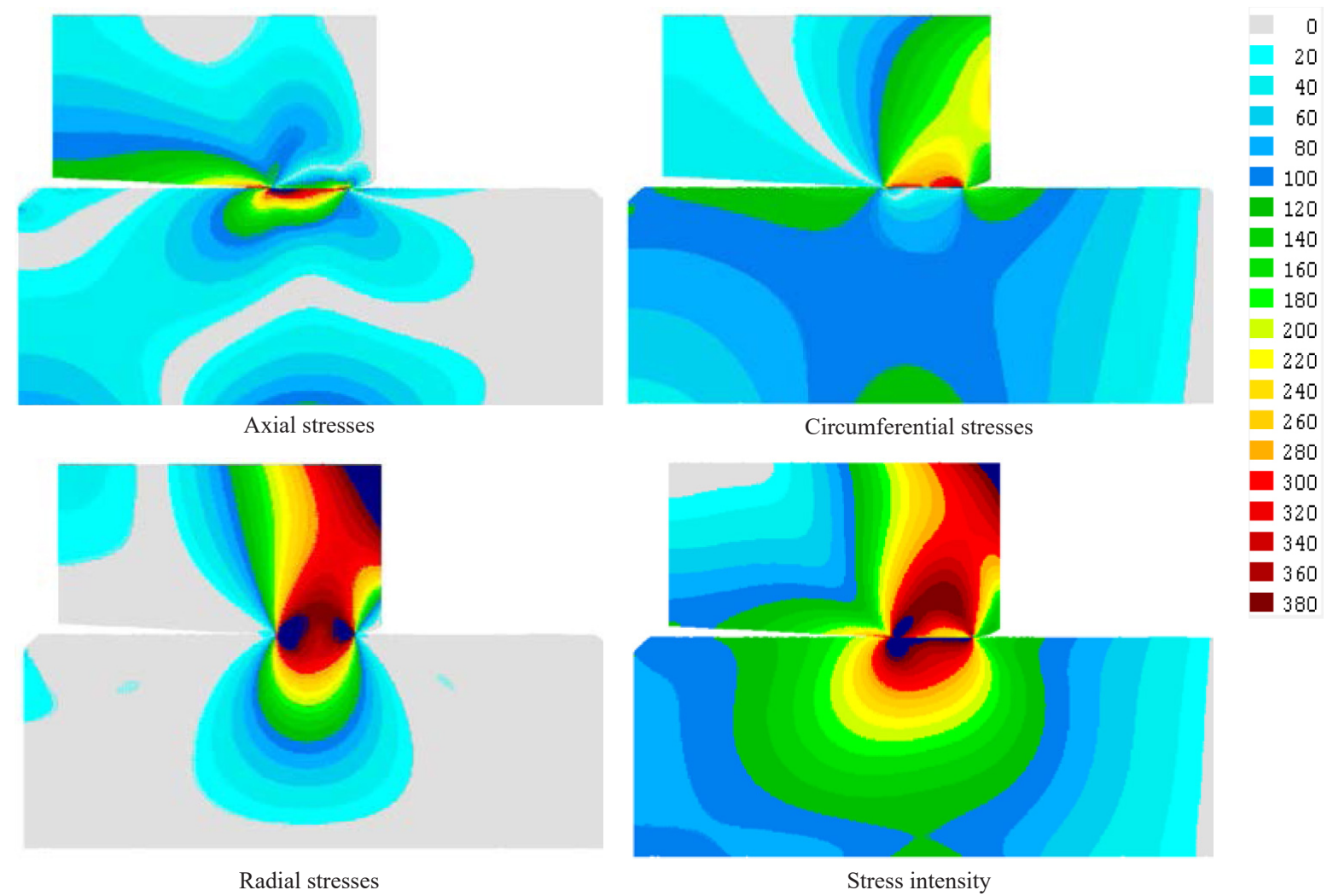

Fig. 4. Stress fields and stress intensities in the workpiece (not taking into account the residual stresses emerging from the inverse cone side) and tool during burnishing (steel $45, \sigma_{s}=360 \mathrm{MPa}, d_{w k p}=45 \mathrm{~mm}$, $D_{w k p} / d_{w k p}=1.64, L=40 \mathrm{~mm}, N_{B}=0.08 \mathrm{~mm}, b=5 \mathrm{~mm}, \alpha=3^{\circ}, \alpha_{1}=10^{\circ}, f=0,12$, compression scheme)

The changes in the friction conditions during burnishing do not influence the regularities of stress distribution in the workpiece along its length and wall thickness, however, their change reflects the level of stresses. When the friction conditions worsen, their growth is observed in all cases. The above-mentioned phenomenon is also revealed at the changes in all the process investigated factors: burnishing interference, tool geometry. At the same time, this growth is insignificant (up to $14 \%$ ) in the investigated ranges of the values of the studied process factors, what confirms the acceptability of averaging the friction forces on the contact surface when solving rheology problems, where the precise analysis of body SSS is not required.

The displacement investigations in the workpiece demonstrated that regardless of the tool position in the hole treated, the maximum displacement in the workpiece, as well as stresses, emerge on its internal surface in radial sections dividing the cylindrical band with front and inverse cones (Fig. 4). When the tool penetrates the workpiece, these displacements have the extreme value from the free end face. At the same time, the hole elastic shrinkage is observed at the mounting end face. During burnishing the value of radial displacements goes down, it reaches its minimum in the workpiece section equidistant from the end faces, and goes up again; when the tool reaches the mounting end face, the displacement extremum shifts to the section dividing the cylindrical band with the front cone. Thus, the workpiece deforms nearer the face ends than in the middle. The same regularity of deformations is also observed along the external surface.

The elastic displacements of the tool in nodal points of its contact with the workpiece are interconnected with the corresponding displacements of its internal surface. Since the general balance of displacements equals the burnishing interference, the least deformation of the tool corresponds to the largest deformation of the workpiece, and vice versa.

For the set burnishing interference the ratio between the workpiece and tool deformations depend not only on the position of the latter in the hole treated, but also on the process factors. 




Fig. 5. Distribution of stresses and stress intensities along the workpiece wall at its loading during burnishing (experiment conditions are the same as for Fig. 4)

It was found out that with the increased width of the tool cylindrical band, the workpiece wall thickness and yield stress, as well as with the decreased angle of the tool front cone, the displacements go down in the workpiece, and go up in the tool.

It is impossible to explain the indicated regularities by the change in the stress level in the workpiece, since the decrease of displacements therein occurs not only with the stress decrease, but also with their growth. It is obvious that there is a more complicated connection between these phenomena, which was established based on the analysis of development of contactless zones of the workpiece deformation during burnishing.

As the experiments carried out before demonstrated [8], when the workpieces are burnished with relative interference exceeding 0.01 (deformation degree is $1 \%$ ), these zones appear in the regions of the front cone, as well as the cylindrical band and inverse the cone of the tool. When the deformation degrees are under $1 \%$, the workpiece contactless deformation occurs only in the region of the tool front cone.

The performed numerical investigation of the workpiece SSS during burnishing with interference up to $0.28 \mathrm{~mm}$ (maximum deformation degree is $0.6 \%$ ) fully confirmed the fact theoretically. Moreover, the results obtained allow affirming that the burnishing process is also accompanied by the contactless elastic deformation of the tool (Fig. 6).

As a result of contactless deformations, the actual width of the contact forming the front cone of the tool with the workpiece $1_{C . A}$ is always less than theoretical one $1_{\text {C.T }}$. It is established that the maximum displacements on the workpiece internal surface are defined not only by the stress level, but also by the actual width of its contact with the tool front cone. The contact width, in turn, depends on the values of burnishing process factors, thus complicating its connection with stresses and displacements.

At the set burnishing interference the contact width goes up with the increased workpiece wall and stresses in it. With the growth of the forming cylindrical band of the tool and yield stress of the material treated, the contact width decreases with the drop of the stress level inside the workpiece. Both in the first and in the second case, the displacement value on the internal surface of the workpiece decreases during its loading. The increase in the angle of the tool front cone also decreases the contact width, however, this occurs with the growth of stresses and displacements in the workpiece.

The change in the friction conditions during burnishing with the relative interference up to 0.6 does not lead to the change in the contact width, however, it influences the displacements of the contact nodal points of the tool with the workpiece. With the improved friction conditions the value of these displacements goes down.

The loading character of the workpiece and displacements in it during burnishing are directly connected with the value of diametric residual deformation on internal and external surfaces. It is established that with the decrease of dis- 


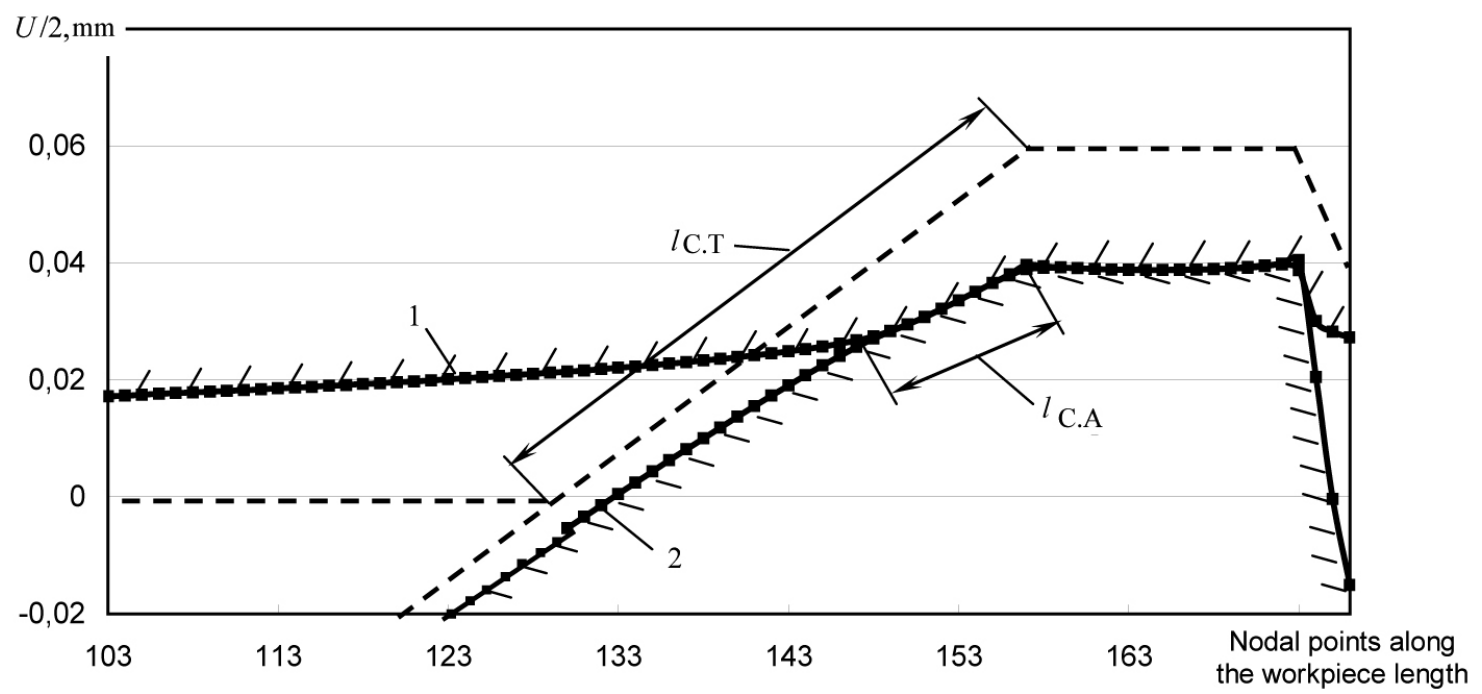

Fig. 6. Contactless deformations $U$ of internal surface of the workpiece 1 and external surface of the tool 2 in the region of the front cone during burnishing (steel $45, \sigma_{\mathrm{s}}=360 \mathrm{MPa}, \mathrm{d}_{\mathrm{wkp}}=45 \mathrm{~mm}, \mathrm{D}_{\text {wkp }} / \mathrm{d}_{\text {wkp }}=1.64, L=40 \mathrm{~mm}$, $\mathrm{N}_{\mathrm{B}}=0.12 \mathrm{~mm}, b=2 \mathrm{~mm}, \alpha=1^{\circ}, \alpha_{1}=10^{\circ}$, compression scheme): $1_{\mathrm{C} . \mathrm{T}}$ and $1_{\mathrm{K} . \mathrm{A}}$ - theoretical and actual width of the contact between the tool and the workpiece

placements in the workpiece during its loading, the value of diametric residual deformation after burnishing decrease as well. This agrees with the results of the experimental investigations performed before, demonstrating that at the set interference of burnishing the value of radial residual deformation goes down with the decreased angle of the tool front cone, increased workpiece wall thickness and yield stress of the material treated, as well as improved friction conditions between the tool and the workpiece.

\section{CONCLUSIONS}

1. The fact of considerable stress concentration in the workpiece in the place of its contact with cylindrical band of the tool was theoretically established as a result of the conducted numerical investigation. The maximum stress level is accrued to the workpiece radial sections going through the tool sections dividing its cylindrical band with front and inverse cones. The increase in the cylindrical band width or radius of its transition to conical regions results in the significant stress decrease and can be considered as a designing method for controlling the level of residual stresses in the workpiece.

2. The maximum stress intensity occurs in the workpiece surface layer, it depends on bur- nishing interference, tool geometry, workpiece wall thickness and is determined by the ability of the material treated for deformation strengthening. The possibility to calculate the stress intensity with the numerical method in different nodal points along the workpiece wall thickness allows theoretically forecasting the cold-work strengthening degree and the depth of surface layer strengthening. The burnishing interference (workpiece deformation degree) is the most important factor influencing the strengthening depth.

3. Worsening of the friction conditions between the tool and the workpiece (in the conducted numerical investigation the friction coefficient increases from 0 up to 0.26 ) at different combinations of the process factors $\left(\mathrm{N}_{B}, b\right.$, $\left.\alpha, \alpha_{1}, \mathrm{D}_{\mathrm{wkp}} / \mathrm{d}_{\mathrm{wkp}}, \sigma_{\mathrm{s}}\right)$ results in the insignificant increase of the stress level in the workpiece (up to 14\%). This demonstrates the admissibility and legitimacy of averaging the friction forces on the contact surface when solving the problem of metal shaping, in which the precise analysis of the workpiece SSS is not required and it is sufficient to have the effort definition useful for deformation, change of shape and dimensions of the body deformed. In the processes of precise treatment, including gauge burnishing, the boundary conditions when investigating the workpiece SSS 
should be formed taking into account the variable (non-stationary) friction conditions.

4. The displacement investigation in the workpiece and tool during burnishing demonstrated the inadmissibility of using the assumption of the tool absolute rigidity in the previous investigations of the workpiece deformed state. It is established that in the total balance of displacements of the workpiece internal surface and tool external surface, the elastic deformations of the latter sufficiently decrease (down to 50\%) the actual burnishing interference. The value of this decrease depends on the tool position in the hole treated, as well as the process factors. For the correct forecasting of stresses in the workpiece its calculation is only possible based on the application of numerical methods.

5. The availability of contactless deformation of the workpiece is theoretically confirmed in the region of the tool front cone, and the elastic contactless deformation of the tool itself is revealed. It is demonstrated that the change in the actual contact width is directly connected with the change in the actual burnishing interference.

6. The improvement of the friction conditions during burnishing decreases the value of diametric residual deformation of the workpiece along the internal surface. This creates the prerequisites to control the dimensional and geometric accuracy of workpieces due to the application of different methods of friction regulation.

\section{Acknowledgements}

This work is a part of project KEGA 014STU$4 / 2015$. The article was supported by the VEGA $1 / 0477 / 14$ project "Research of influence of selected characteristics of machining process on achieved quality of machined surface and problem free assembly using high Technologies" supported by the scientific grant agency of the Ministry of Education of the Slovak Republic and of Slovak Academy of Sciences. This publication is the result of implementation of the project: "University Scientific Park: Campus MTF STU - CAMBO" (ITMS: 26220220179) supported by the Research \& Development Operational Program funded by the EFRR.

\section{REFERENCES}

1. Sivtsev N.S. Mechanical and mathematical model for loading the local contact at SPD under non stationary friction conditions. Mechanical engineering, 10, 2004, 3-12.

2. Makushok, E.M., Kalinovskaya, T.V., and Belyi, A.V., Massoperenos v protsessakh treniya (Mass Transfer in Friction Processes), Minsk: Nauka i Tekhnika, 1978.

3. Makushok E.M., Kalinovskaya T.V., Krasnevskya S.M. et al. Fundamentals of the processes of surface plastic deformation. Science and engineering, 1988.

4. Božek P. and Pivarčiová E. Flexible manufacturing system with automatic control of product quality. Strojarstvo, 55(3), 2013, 211-221.

5. Birger I.A., Shorr B.F. and Iosilevich G.B. Calculation of machine part strength: Referencebook. Mechanical engineering, 1979.

6. Božek, P. Automated detection type body and shape deformation for robotic welding line. Advances in Intelligent Systems and Computing, 240, 2014, 229-240.

7. Abramov I.V., Fattiev F.F., Dulotin V.A. et al. High-tension fits with guaranteed interference. Izhevsk: ISTU Publishers, 2002.

8. Proskuryakov Y.G. Hole burnishing. Sverdlovsk: Mashgiz, 1961.

9. Božek P., Pokorný P. Analysis and evaluation of differences dimensional products of production system. Applied Mechanics and Materials, 611, 2014, 339-345.

10. Rozenberg A.M., Rozenberg O.A. Mechanics of plastic deformation in the processes of cutting and deforming broaching. Kiev, Naukova dumka, 1990.

11. Monchenko V.P. Effective technology of manufacturing hollow cylinders. Mechanical engineering, 1980.

12. Yanchenko I.I. Investigation of the burnishing process under different conditions of the tool lubricating: Thesis of Candidate of Technical Sciences. Izhvesk. 1973.

13. Korshunov A. The generalized model of valuation of constructive-technological complexity of a machine-building item. Pollack Periodica, 2(3), 2007, 135-146.

14. Tarasov V.V. Development of scientific bases for manufacturing the thin-wall parts by axisymmetric deformation of rotational tool: Thesis of Doctor of Technical Sciences. Izhevsk, 1999.

15. Debski H., Koszalka G. and Ferdynus M. Application of FEM in the analysis of the structure of a trailer supporting frame with variable 
operation parameters. Eksploat. i Niezawodn. Maint. Reliab., 14, 2012, 107-113.

16. Garbacz T., Jachowicz T., Gajdoš I., Kijewski $\mathrm{G}$. Research on the influence of blowing agent on selected properties of extruded cellular products. Advances in Science and Technology Research Journal, 2015, 9(28), 81-88.

17. Peterka P., Krešák J., Kropuch S., Fedorko G., Molnar V. and Vojtko M. Failure analysis of hoisting steel wire rope. Engineering Failure Analysis, 45, 2014, 96-105.

18. Molnár V., Boroška J. and Dečmanová J. Mechanical properties of steel rope wires - quality test assurance. Acta Montanistica Slovaca, 15, 2010, 23-30.

19. Molnár V., Fedorko G., Krešák J., Peterka P. and Fabianová J. The influence of corrosion on the life of steel ropes and prediction of their decommissioning. Engineering Failure Analysis, 74, 2017, 119-132.

20. Kmet S., Stanova E., Fedorko G., Fabian M. and Brodniansky J. Experimental investigation and finite element analysis of a four-layered spiral strand bent over a curved support. Engineering Structures, 57, 2013, 475-483. 